\title{
Taxonomy of $\kappa$ : A Review of Definitions and Estimation Approaches Targeted to Applications
}

\section{by Olga-Joan Ktenidou, Fabrice Cotton, Norman A. Abrahamson, and John G. Anderson}

\section{INTRODUCTION}

In a way perhaps not dissimilar to stress drop (Atkinson and Beresnev, 1997), the high-frequency attenuation parameter $\kappa$ (kappa), introduced by Anderson and Hough (1984), is one of the most used yet least understood or agreed-upon parameters in engineering seismology. It describes the deviation at high frequencies between observed Fourier amplitude spectra calculated from seismograms and an $\omega^{-2}$ source model, such as the Brune (1970) model. Almost 30 years after its introduction, $\kappa$ is used by seismologists and engineers alike and constitutes an important input parameter for several applications. Perhaps because of its importance, it is estimated, physically explained, and used in many different ways. This note aims to illustrate the multiple approaches to its estimation, and to suggest that, in order to reduce ambiguities, the parameter should always be given a notation consistent with its measurement and application to help avoid inconsistencies in its application of $\kappa$ scaling to ground-motion models.

Hanks (1982) observed that above a given frequency the acceleration spectrum decays sharply. He termed this frequency $f_{\max }$ (e.g., Fig. 1a) and attributed it mainly to local site conditions. Soon after, Anderson and Hough (1984) introduced an alternative parameter to model this decay, which is the one most commonly used today: $\kappa$. They measured $\kappa$ directly from the high-frequency part of the acceleration Fourier amplitude spectrum of a record. Above a certain frequency (which they named $f_{\mathrm{e}}$ but we will call here $f_{1}$ ), the overall shape of the spectrum generally decays exponentially with frequency; the decay constant is most easily measured by finding a linear approximation to the spectrum plotted in log-linear space. The slope of the linear approximation is $-\pi \kappa$ (e.g., Fig. 1b). In this note we use the notation $\kappa_{\mathrm{r}}$ for individual observations of $\kappa$, for example, the $\kappa$ value corresponding to the slope of a particular record; this record may be at any epicentral distance $R_{\mathrm{e}} \geq 0$. Anderson and Hough (1984) also observed that $\kappa_{\mathrm{r}}$ at individual stations increases with distance and concluded that it includes components related both to the local geology of the top few $\mathrm{km}$ of crust beneath the station and to the regional structure. They then suggested that the site component of $\kappa$ (denoted $\kappa_{0}$ ) could be computed by extrapolating the $\kappa_{\mathrm{r}}$ values to zero epicentral distance, thus correcting for the regional effect of anelastic $Q$.

In this note, we discuss the use of $\kappa_{0}$ in various engineering seismology applications today and why interest in this parameter has been revived. We briefly discuss its possible physical interpretations, and detail the known approaches to estimate $\kappa_{0}$ from seismic records. We group these approaches into families according to basic features, such as the range of frequencies over which $\kappa_{0}$ is computed and the trade-off with path effects. We then discuss the alternative option for estimating $\kappa_{0}$ when site-specific records are not available, based on empirical correlations with $V_{S 30}$. We collect previously published correlations and demonstrate the scatter observed across different studies. Finally, we make suggestions as to how $\kappa_{0}$ estimation can be made in a more consistent way with the applications that use it, and how existing correlations can be made more consistent to improve both the inference of $\kappa_{0}$ in the absence of site-specific data and the physical understanding of $\kappa_{0}$.

\section{$\kappa:$ A MULTITUDE OF APPLICATIONS, PHYSICAL INTERPRETATIONS, AND MEASUREMENT METHODS}

\section{Importance of $\boldsymbol{\kappa}_{0}$ and Fields of Application}

Over the last decades, $\kappa$ has been used in different applications. In source studies, removing the attenuation term is fundamental in order to study self-similarity of the source spectrum; this can be done by inverting for the theoretical $Q$, using empirical Green's functions (EGFs) or multiple EGFs to remove path and site effects, measuring spectral parameters from the coda, or using $\kappa_{\mathrm{r}}$ to correct for high-frequency attenuation (e.g., Irikura, 1986; Abercrombie, 1995; Mayeda and Walter, 1996; Hough, 1997; Lancieri et al., 2012). In the generation of synthetic ground motion using point-source or finite-fault stochastic or hybrid simulation approaches, $\kappa_{0}$ is applied as a low-pass filter to constrain high frequencies, affecting peak ground motion and spectral shape (Boore, 1986; Beresnev and Atkinson, 1997; Boore, 2003; Graves and Pitarka, 2010). Even in physicsbased simulations using theoretical Green's functions (TGFs; e.g., Zeng et al., 1994; Graves, 1996; Halldorsson and Papageorgiou, 
(a)

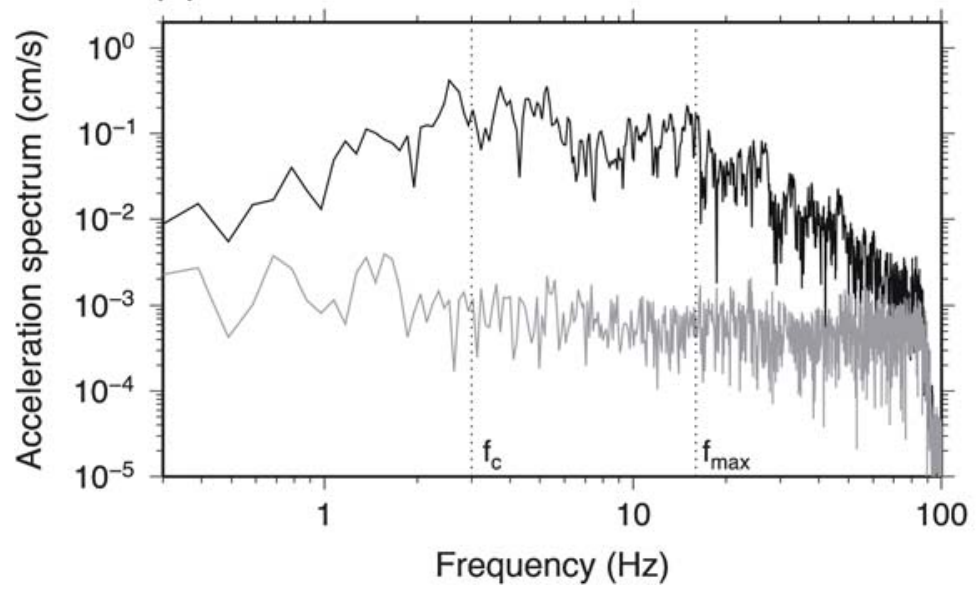

(b)

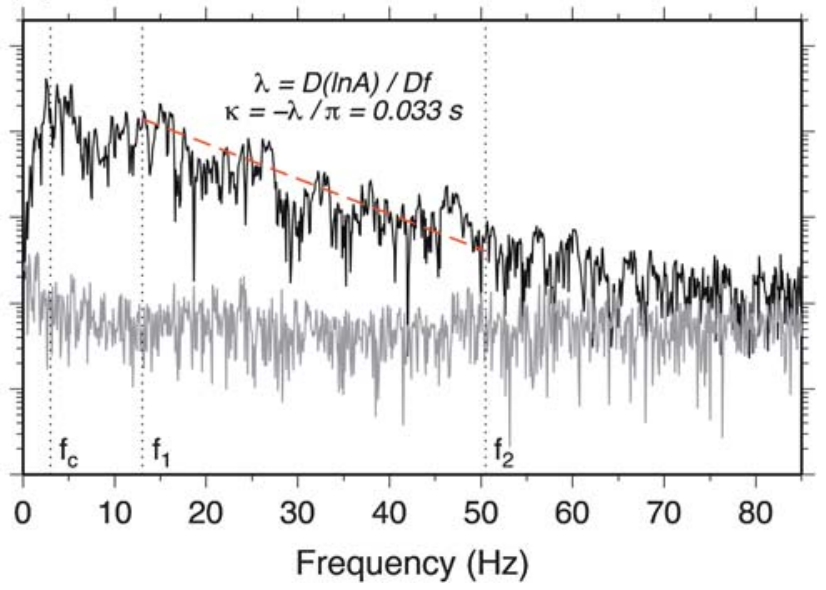

$\Delta$ Figure 1. (a) Definition of $f_{\max }$ as the onset of the crashing of the $S$-wave spectrum (solid black line), plotted on a log-log scale. Also shown are the corner frequency and the noise spectrum (solid gray line). (b) Definition of $\kappa_{\mathrm{r} \_ \text {As }}$ as the slope of the spectrum in the frequency range $\left(f_{1}-f_{2}\right)$ in log-linear space.

2005; Mai et al., 2010; Schmedes et al., 2010; Foster et al., 2012), imposing such a constraint is necessary to achieve realistic results at high frequencies. The amount of the $\kappa$ correction in such approaches depends on how $Q$ is incorporated in the TGFs. In the creation and calibration of ground-motion prediction equations (GMPEs) based on stochastic simulations, near-surface attenuation is implicitly considered through a set of $\kappa_{0}$ values considered applicable to the region (e.g., Toro et al., 1997).

Recently, the use of $\kappa$ in the engineering seismology community has been revived. On the one hand, the need has arisen to adjust GMPEs from host to target regions, often from active regions with soft rock to less active regions with hard rock (Delavaud et al., 2012) with approaches such as the hybrid empirical method of Campbell $(2003,2004)$. The scaling from soft to hard rock is made considering the differences in $V_{S 30}$ and $\kappa_{0}$ to account for both site amplification that is dominant at lower frequencies, and site attenuation that dominates high frequencies (Cotton et al., 2006; Van Houtte et al., 2011). Moreover, in probabilistic seismic hazard assessment (PSHA) for critical facilities, it is common to conduct site-specific site response analyses. This requires adjusting GMPEs, even within the same region, to bedrock level and then computing site response up to the surface. Adjusting the GMPEs to hard-rock conditions requires knowledge of $\kappa_{0}$. Indeed, the Probabilistic Seismic Hazard Analysis for Swiss Nuclear Power Plant Sites (PEGASOS) Refinement Project (Biro and Renault, 2012) showed that, for hard rock, the $V_{S 30}$ and $\kappa_{0}$ corrections can lead to differences up to a factor of 3 in the high-frequency part of the response spectrum. Given the significance of $\kappa_{0}$, in the past year attempts were also made to explicitly include it in the functional form of GMPEs (Laurendeau et al., 2013), an idea that actually originated as early as Anderson et al. (1996).

\section{Physical Interpretations: Source, Path, and Site Components}

Since the 1980s, there have been debates as to the origins of $\kappa$. The difficulty of mapping $\kappa$ onto physical parameters was predicted and explained by Hanks (1982) even at the time of introducing $f_{\text {max }}, \kappa^{\prime}$ s predecessor, to model what he then called "the crashing spectrum syndrome." According to him, the catch lies in the fact that it is the absence of signal that is to be interpreted, not its definitive presence. Before the definition of $\kappa$ or $f_{\max }$, several observations showed an apparent departure from the scaling law of Aki (1967) for small-magnitude events, that is, the observed source corner frequency, $f_{c}$, ceased to increase for decreasing magnitudes. This masking of the expected $f_{c}$ had been attributed to source-related effects (Bakun et al., 1976), to anelastic and scattering site attenuation (Frankel, 1982), or to both (Archuleta et al., 1982). Then Hanks (1982) and, later on, Anderson (1986) attributed the newly defined $f_{\max }$ to site attenuation. This is the prevalent view today, though some studies have again related it to source properties, such as the width of the ruptured fault zone (Papageorgiou and Aki, 1983; Aki, 1987; Papageorgiou, 1988; Gariel and Campillo, 1989; Papageorgiou, 2003; Tsurugi et al., 2008; Iwakiri and Hoshiba, 2012; Wen and Chen, 2012).

To enable discussion for the origins of $\kappa_{\mathrm{r}}$, it is useful to start with a general model encompassing all of the various hypotheses. In equation (1), $\kappa_{0}$ is the site component, $\kappa_{\mathrm{s}}$ is introduced to represent the source contribution, and $\tilde{\kappa}\left(R_{\mathrm{e}}\right)$ follows the notation of Anderson (1991) for a generalized distance dependence on the epicentral distance $R_{\mathrm{e}}$ :

$\kappa_{\mathrm{r}}=\kappa_{0}+\kappa_{\mathrm{s}}+\tilde{\kappa}\left(R_{\mathrm{e}}\right)$

Today, most studies model $\kappa_{\mathrm{r}}$ as a site and path effect. Datasets with adequate stations and events can also resolve 
source contributions, i.e., $\kappa_{\mathrm{s}}$ (Tsai and Chen, 2000; Purvance and Anderson, 2003; Van Houtte et al., 2011; Kilb et al., 2012). Many of these studies find that $\kappa_{s}$ is mostly related to the scatter of $\kappa_{\mathrm{r}}$ measurements.

In equation (1), the constraint on the distance dependence is that $\tilde{\kappa}(0)=0$. As expected from studies that find regional variability in $Q$, there are variable results for the functional form of the distance dependence and importance of this term. The increase of $\kappa_{\mathrm{r}}$ with distance has been explored in a number of studies. The simplified initial assumption of Anderson and Hough (1984) for linear distance dependence mostly served an illustrative purpose. Notwithstanding, it has proved a reasonable approximation for several datasets (Nava et al., 1999; Douglas et al., 2010; Gentili and Francheschina, 2011; Ktenidou et al., 2013). Other studies find distance dependencies that deviate in different ways from the initial linear assumption (Hough and Anderson, 1988; Hough et al., 1988; Anderson, 1991; Humphrey and Anderson, 1992; Castro et al., 1996; Fernández-Heredia et al., 2012). Some studies have even found negligible distance dependence in some regions out to $80 \mathrm{~km}$ (Tsai and Chen, 2000; Purvance and Anderson, 2003).

In summary, then, the literature on studies that have tried to explain the physical processes behind $\kappa$ is consistent with the model expressed in equation (1), with site conditions having the key role, and source and distance terms having variable importance depending on the region and study. In what follows, we focus on $\kappa_{0}$, the site parameter of $\kappa_{\mathrm{r}}$, which is the most widely accepted point of view.

\section{Measuring $\kappa$ from Seismic Records: Types of Approaches}

People measure $\kappa$ in different ways. In Table 1, we outline some of the main approaches for estimating $\kappa_{0}$. Naturally, more examples can be found in the literature, but our aim is to create a relatively small number of approaches, or rather families of approaches, based on certain common features, such as the principle behind the approach, the frequency range over which $\kappa$ is computed, and how the distance dependence and the tradeoff with $Q$ are dealt with.

We make a clear distinction between $\kappa_{\mathrm{r}}$ and $\kappa_{0}$. Some of the approaches to measure $\kappa_{0}$ start with individual measurements of $\kappa_{\mathrm{r}}$ (i.e., observations on individual spectra at some distance $r$ ), which must then be combined and extrapolated to zero distance to obtain an estimate of $\kappa_{0}$ for the site. Others yield directly the $\kappa_{0}$ (i.e., the site-specific, zero-distance $\kappa$ derived from many observations). There are different ways of extrapolating $\kappa_{\mathrm{r}}$ values to zero distance. A simplified approach is to assume a linear dependence of $\kappa_{\mathrm{r}}$ with distance and perform a standard linear regression. Considerations of seismic ray theory show that this assumes that $Q$ and the shear velocity are independent of depth. A more general approach is that suggested based on better data by Hough and Anderson (1988) and Anderson (1991), in which the only constraint is that $\kappa_{\mathrm{r}}$ is a smooth function of distance to be determined by the data, or any nonparametric regression allowing for more realistic underlying $Q$ structures. We will proceed to describe the main families of approaches for $\kappa$ estimation.

- Acceleration Spectrum: Following the classic definition of Anderson and Hough (1984), $\kappa_{\mathrm{r}}$ can be directly measured in log-linear space on the high-frequency part of the Fourier acceleration spectrum of the $S$ waves, above $f_{1}$ (Fig. 1b). We will refer to this original definition as $\kappa_{\mathrm{r} \_A s}$. Because a component of horizontal wave propagation, affected by $Q$, is present in these measurements, an extrapolation to zero distance (assuming frequency-independent $Q)$ will lead to the site-specific attenuation component, $\kappa_{0 \_ \text {AS }}$. This approach can only be used for relatively large event magnitudes, as $f_{1}$ must exceed $f_{c}$ to avoid any tradeoff with the source. $f_{1}$ is visually picked. It is less than $f_{\max }$ according to Anderson and Hough (1984), whereas Anderson (1986) proposes a more rigorous alternative: $f_{95}$, at $95 \%$ of the spectrum energy. Tsai and Chen (2000) suggest $f_{1}$ is similar to the $f_{\text {max }}$ defined by Hanks and McGuire (1981): $f_{\max }=Q \beta / \pi R$ and which can be rewritten as: $f_{\max }=1 / \pi \kappa$ according to Anderson (1986), if $Q$ is independent of frequency.

- Transfer Function: A variation of the classic method was proposed by Drouet et al. (2010), in which the site-specific $\kappa_{0}$ can be measured directly from the high-frequency part of the site transfer function that has been derived through source-path-site inversions on a set of records from various stations and events. This $\kappa_{0}$ we will call $\kappa_{0 \_ \text {TF }}$. Here the measurement frequency range is above the resonant peak and any higher mode amplification peaks, and the transfer function is computed using one of the stations as reference, ignoring its site amplification and attenuation. Similarly, Frankel et al. (1999) measured $\kappa$ from surface-to-reference amplification functions relative to an ideal reference site. Motazedian (2006) and Ghofrani et al. (2013), on the other hand, applied a similar method to the horizontal-to-vertical spectral ratio, that is, using the vertical component as reference. In those approaches, for a non-zero reference $\kappa$, the measured quantity was the difference between horizontal and vertical $\kappa$.

- Source Spectrum: Another variation of the classic method is the one suggested by Oth et al. (2011), who measure $\kappa_{\mathrm{r}}$ as the high-frequency decay on the Fourier source spectrum, which is actually the Fourier amplitude from the source at depth, recorded at the surface. Their approach first removes the effect of $Q(f)$ to a reference distance of $5 \mathrm{~km}$ (where its effect can practically be ignored) and then corrects for the site amplification, without, however, including any high-frequency decay therein. Hence, this decay can be found in the derived source spectra (we name it $\left.\kappa_{\mathrm{r}_{-} S S}\right) . \kappa_{\mathrm{r}_{-} S S}$ corresponds to a virtually zero-distance estimate of $\kappa_{0}$ and no extrapolation is necessary. But because we derive one value per event, we must average all values available at each site to estimate the site's overall $\kappa_{0 \_}$ss. A similar approach is used by Margaris and Boore (1998), correcting for both site amplification and whole-path attenuation before deriving $\kappa_{0}$. 
Table 1

The Main Families of Approaches Available for Estimating Site-Specific, Zero-Distance $\kappa_{0}$

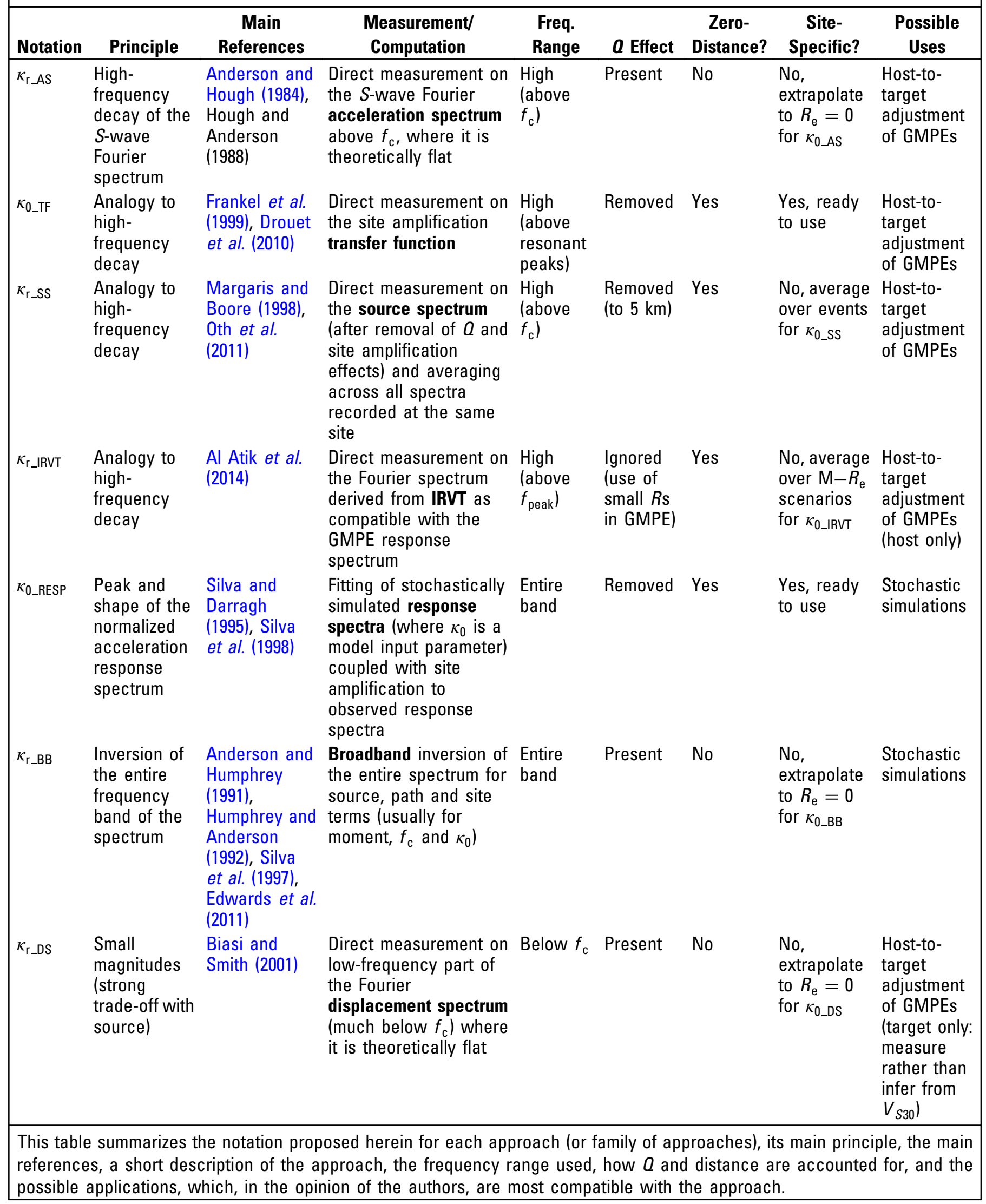


- Inverse Random Vibration Theory: The final variation of the original method discussed here does not use actual records, but response spectra produced by GMPEs for short distances. Al Atik et al. (2014) use inverse random vibration theory and the technique of Rathje et al. (2005) to derive compatible Fourier spectra from a series of response spectra computed through the GMPE formulae for a suite of magnitude and distance scenarios (below $20 \mathrm{~km}$ ). Then $\kappa_{\mathrm{r}_{-} \text {IRVT }}$ can be measured on the high-frequency part of each GMPE-consistent Fourier spectrum, and it is assumed site-specific in as much as the $Q$ effect is considered negligible. However, because one value of $\kappa_{\mathrm{r}_{-} \mathrm{IRVT}}$ is derived for each scenario, these values are averaged over all scenarios to estimate a single $\kappa_{0 \_ \text {IRVT }}$, which may be considered as the GMPE's native or intrinsic $\kappa_{0}$.

- Response Spectrum: In contrast to the classic that relates $\kappa$ to the decay of the high-frequency part of the Fourier spectrum, another approach relates it to the spectral shape of the normalized response spectrum. Introduced by Silva and Darragh (1995) and later used by Silva et al. (1998), this method uses stochastically generated acceleration response spectra (where $\kappa_{0}$ is one of the point-source seismological model input parameters and is applied to the entire frequency range) coupled with the site amplification computed from the site profile. These simulated spectra are then normalized and compared to observed response spectra to compute the input $\kappa$ (let us denote this one $\left.\kappa_{0 \_ \text {RESP }}\right)$ that gives the best fit between them. One important difference from all previous approaches is that, here, the entire frequency band is used for the fitting and not only the high-frequency part. In this approach, trade-offs with stress drop are avoided to a degree, as the response spectra are normalized by peak ground acceleration and then stacked (Hiemer et al., 2011).

- Broadband Inversions: A large family of approaches which again use the entire frequency band, but are not restricted to short distances, are what we will call here the family of broadband inversions. The essence of these methods is the assumption of a source spectral shape, with the objective of extracting $\kappa_{\mathrm{r}}$ when the earthquake corner frequency intrudes into the frequency band used for the measurement. These separate the source, path, and site effects in various ways to yield individual values of what we will denote $\kappa_{\mathrm{r} \_\mathrm{BB}}$. These are then extrapolated to $\kappa_{0 \_\mathrm{BB}}$. One advantage of broadband inversions is that, unlike the classic approach, they are not constrained as much by the event magnitude, meaning they can use more abundant smallmagnitude earthquake data. Numerous broadband inversion schemes can be found in literature. Here we will mention only some of the main schemes. Anderson and Humphrey (1991) invert for $f_{\mathrm{c}}$ (or stress drop), spectral level, and $\kappa_{\mathrm{r}}$, assuming a smooth spectral shape to partly overcome the trade-off with stress drop. Humphrey and Anderson (1992) perform the broadband inversion after removing the empirical or modeled site response from each spectrum. Based on a method by Scherbaum (1990),
Edwards et al. (2011) use a simultaneous broadband inversion of the velocity spectrum resolving for $f_{c}$, moment, and $\kappa_{\mathrm{r} \_\mathrm{BB}}$. Kilb et al. (2012) experiment with fixing the stress drop to a reasonable average value to overcome the trade-off and then invert for moment and $\kappa_{\mathrm{r}-\mathrm{BB}}$. Along the same lines, we may also consider the multiple EGF approaches that reduce the trade-off between $f_{\mathrm{c}}$ and $\kappa_{\mathrm{r}-\mathrm{BB}}$ (Hough, 1997; Frankel et al., 1999; Hough et al., 1999). Finally, we also mention the approach introduced by Silva et al. (1997), which can yield either a site-specific or a site-class-specific $\kappa_{0}$, depending on the site data available. This is a broadband inversion of the $\log$ of the empirical Fourier spectrum, which has been coupled to a smoothed 1D amplification function (derived either from the site profile or the site class); it yields moment and stress drop, and separates $\kappa_{0}$ from the $Q(f)$ model (described by $Q_{0}$ and $\eta$ ).

- Displacement Spectrum: The classic approach uses relatively large magnitudes in order to measure spectral decay above $f_{\mathrm{c}}$. Biasi and Smith (2001) proposed an approach that expands the method to very small magnitudes, where data is much more abundant. These authors measure $\kappa_{\mathrm{r}}$ directly on the Fourier displacement spectrum, keeping much below the (rather high) $f_{\mathrm{c}}$, rather than on the Fourier acceleration spectrum and keeping above the (rather low) $f_{\mathrm{c}}$. Rather than using records from earthquakes, say, above $\mathbf{M} 4$, for which $f_{\mathrm{c}}$ is below $10 \mathrm{~Hz}$, and measuring $\kappa_{\mathrm{r}}$ as the departure of the acceleration spectrum from the horizontal over, say, $10-30 \mathrm{~Hz}$, we can use records from earthquakes with $\mathbf{M}<1$, for which $f_{\mathrm{c}}$ may exceed $70 \mathrm{~Hz}$, and measure $\kappa_{\mathrm{r}}$ as the departure of the displacement spectrum from the horizontal over potentially the same frequency range. One advantage of this approach (other than the abundance of data) is that the theoretical basis for treating the displacement spectrum at the source as flat below $f_{\mathrm{c}}$ is actually stronger than the basis for treating the acceleration spectrum as flat above it, because the latter depends on the validity of the $\omega^{-2}$ assumption. Let us denote individual $\kappa_{\mathrm{r}}$ values thus measured as $\kappa_{\mathrm{r}-\mathrm{DS}}$, and the extrapolated zero-distance site parameter as $\kappa_{0 \_ \text {DS }}$ (sometimes referred to also as $\kappa_{0 \_ \text {mini }}$ ).

\section{Inferring $\kappa_{0}$ from $\kappa_{0}-V_{S 30}$ Correlations}

The previous section discussed the measurement of $\kappa_{0}$ from data. In practice, when site-specific data is not available (for instance, when adjusting a GMPE to a stable continental region with little seismic activity and instrumentation), $\kappa_{0}$ is often inferred from available site data, namely $V_{S 30}$ (Biro and Renault, 2012). For this reason, we now revisit existing $\kappa_{0}-V_{S 30}$ correlations and discuss their scatter and applicability. Given that $\kappa_{0}$ is the site component of $\kappa_{r}$, it is reasonable to expect that harder sites will have lower attenuation, and thus $\kappa_{0}$ will decrease as shear-wave velocity increases. Moreover, as $V_{S 30}$ is often available, this has led to various investigations of the correlation between $\kappa_{0}$ and $V_{S 30}$ values (Fig. 2a). However, the scatter observed in such correlations is large and the correlation of $\kappa_{0}$ with $V_{S 30}$ becomes clear only when we compare $\kappa_{0}$ across several site 
(a)

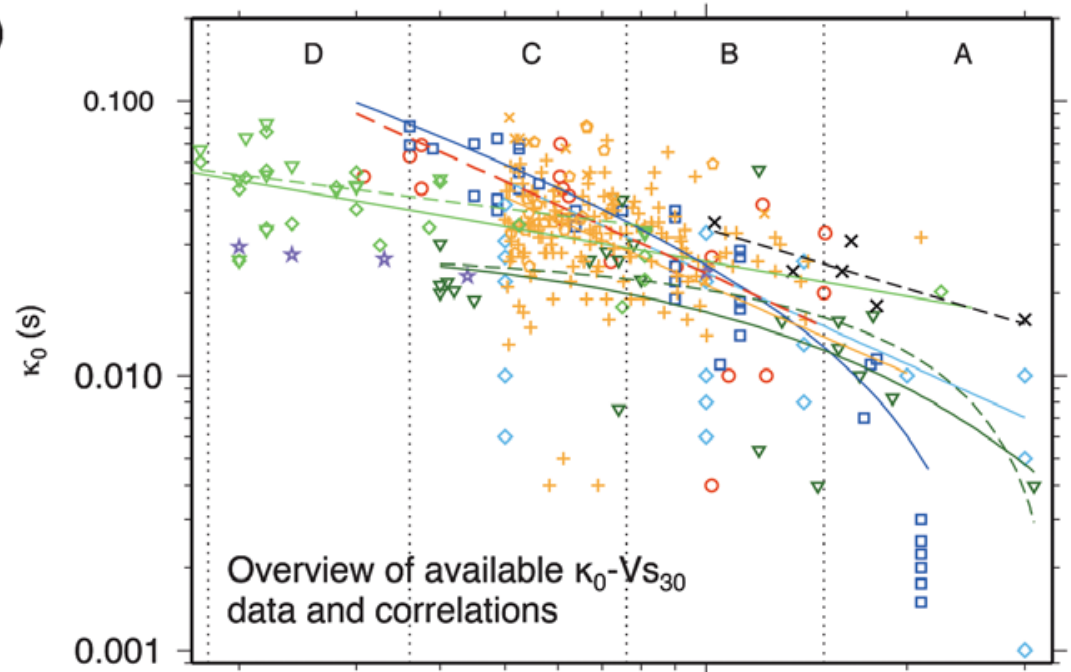

- Silva et al., 1998 (Calif. - $K_{\text {RESP }}$ ) --

口 Chandler et al., 2006 (global - $K_{\text {RESP }}$ ) -

$\diamond$ Drouet et al., 2010 (France - $\mathrm{K}_{\mathrm{AS}}$ ) -

$\nabla$ Edwards et al., 2011 (Switzerland - $\mathrm{K}_{\mathrm{BB}}$ ) linlin - linlog - -

Van Houtte et al., 2011 (Japan - $\mathrm{K}_{\mathrm{AS}}$ ) Van Houtte et al., 2011 (Calif. - $\mathrm{K}_{\mathrm{AS}}$ ) Van Houtte et al., 2011 (Taiwan - $\mathrm{K}_{\mathrm{AS}}$ ) Ktenidou et al., 2013 (Greece - $\mathrm{K}_{\mathrm{AS}}$ )

Ktenidou et al., 2012a (Greece - $\mathrm{K}_{\mathrm{AS}}$ ) -

$\times$ Ktenidou et al., 2012a (Greece - $\mathrm{K}_{\mathrm{TF}}$ )

$\nabla \quad$ Ktenidou et al., 2012b (Switzerland - $\mathrm{K}_{\mathrm{AS}}$ ) - -

(b)

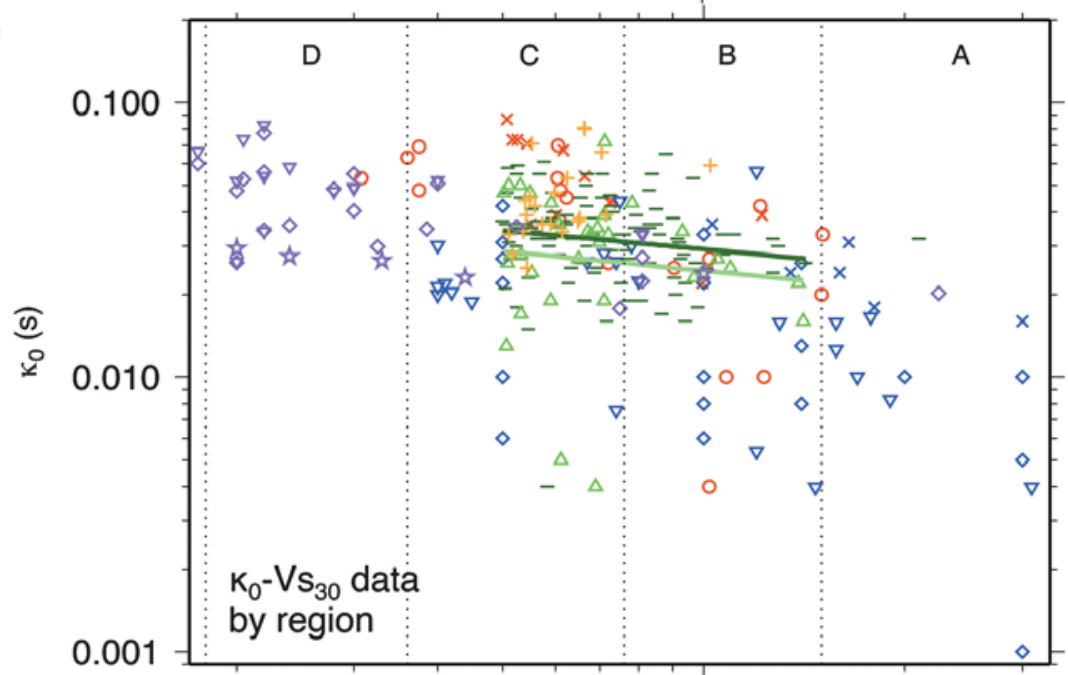

California:

Silva et al., 1998; Van Houtte et al., 2011

Switzerland-France:

Drouet et al., 2010; Edwards et al., 2011;

Ktenidou et al., 2012b

Japan - high Q zone:

Van Houtte et al., 2011

Japan - low Q zones:

Van Houtte et al., 2011

Taiwan:

Van Houtte et al., 2011

Greece:

Ktenidou et al., 2013; 2012a

(c)

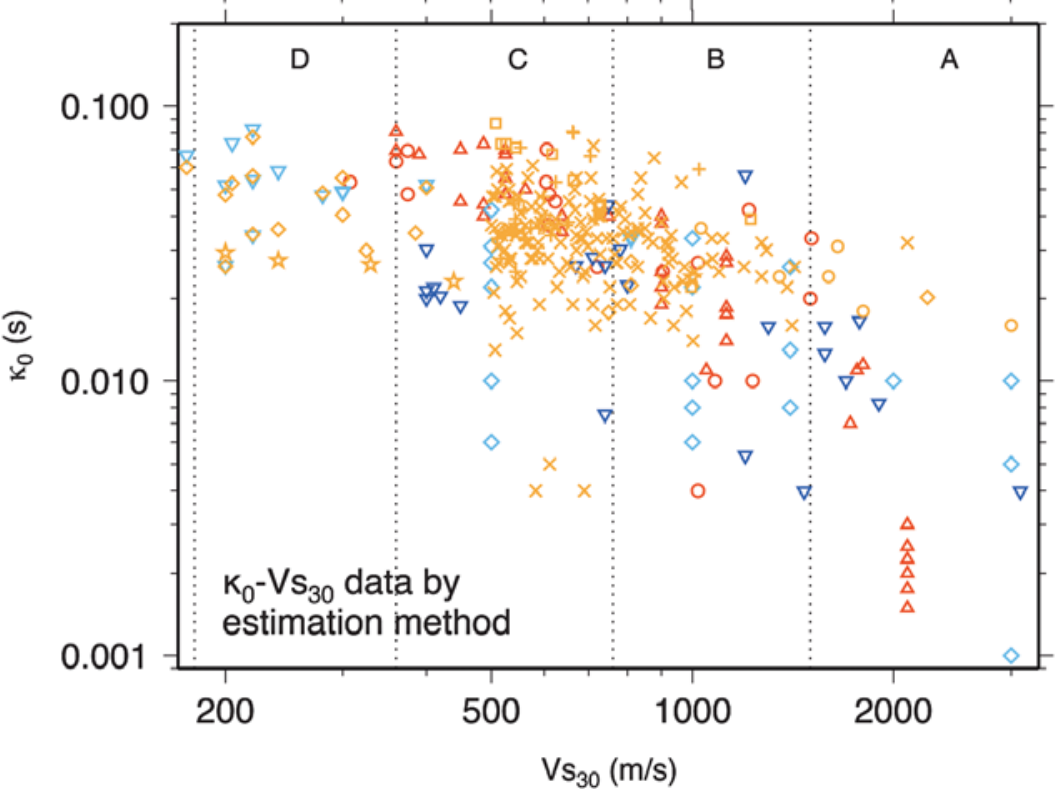

K_RESP:

Silva et al., 1998; Chandler et al., 2006

K_TF:

Drouet et al., 2010; Ktenidou et al., 2012a

K_BB:

Edwards et al., 2011

K_AS:

Van Houtte et al., 2011;

Ktenidou et al., 2013; 2012b

$\Delta$ Figure 2. (a) Existing $\kappa_{0}-V_{S 30}$ correlations proposed in the literature. (b) Grouping of existing correlation data with region. (c) Grouping of existing correlation data with method. Also shown are the limits of site classes $A$ through $E$ according to the National Earthquake Hazards Reduction Program (NEHRP) (see BSSC, 2003). 
classes, say for a $V_{S 30}$ range of $360-1500 \mathrm{~m} / \mathrm{s}$ (i.e., categories C through A of the National Earthquake Hazards Reduction Program [NEHRP], Building Seismic Safety Council [BSSC], 2003; see Fig. 2a). This large degree of scatter should come as no surprise, for several reasons.

First, $V_{S 30}$ is only a proxy for the deeper $V_{S}$ profile that controls local site amplification. Its adequacy as a proxy for site attenuation, which is tied to the first few $\mathrm{km}$ of the profile (Anderson and Hough, 1984; Campbell, 2009), is questionable. From a physical viewpoint, we would also expect other parameters to correlate to $\kappa_{0}$, possibly tied to the deeper structure. One such example is the depth to bedrock, recently found to correlate to $\kappa_{0}$ to a similar degree as does $V_{S 30}$ (Ktenidou et al., 2012).

The second reason is the scatter present in the $V_{S 30}$ values themselves, resulting from the method of measuring (or, at worst, inferring) the $V_{S}$ profile for each site. Moss (2008) and Boore (2006) find differences of 30\%-50\% in the derived $V_{S 30}$ between different methods. For instance, $V_{S}$ can be measured from invasive methods (such as crosshole and downhole geophysics, $P-S$ suspension logger, cone penetration tests [CPT]) or noninvasive methods (active approaches, such as multichannel analysis of surface waves [MASW], or passive, such as spatial autocorrelation [SPAC] and $f-k$ ). Scatter in $V_{S 30}$ is a considerable source of uncertainty but lies outside the scope of this note.

The third reason is the heterogeneity in the $\kappa_{0}$ values themselves. Van Houtte et al. (2011) combined all existing correlations starting from the pioneering work of Silva et al. (1998) and up to their own recent analyses to derive global correlations. The data retrieved are heterogeneous in several ways:

1. Regions: First, the data come from different regions around the world. It is possible that even for similar sites, quite large regional differences in $\kappa_{0}$ may exist due to regional differences in $Q$ in the shallow crust (Boore and Joyner, 1997). Atkinson (1996) observed this for hard rock sites in eastern and western Canada. Chandler et al. (2006), who used a global dataset to derive their correlations, admit their scatter is partly due to the variability of the underlying crustal $Q$ and $V_{S}$ profile for similar $V_{S 30}$ values.

2. Methods: The studies that derived these $\kappa_{0}$ values use a variety of methods: from the classic method of direct measurement on the high-frequency part of Fourier spectra (Douglas et al., 2010; Edwards et al. 2011; Van Houtte et al., 2011), to the measurement on the tail of the site transfer function (Drouet et al., 2010), to the fitting of stochastic point-source simulations to observed response spectra (Silva et al., 1998), to simultaneous broadband inversion of the velocity spectrum (Edwards et al., 2011). Aside from this between-method scatter, one may also consider the possible within-method scatter, due to the different ways users may apply the same method (see e.g., Ktenidou et al., 2013, for an illustration of the strong possible variability within the classic method).

3. Range of Frequencies: Considering that the data in the different $\kappa_{0}-V_{S 30}$ correlations come from a period of over two decades, we expect instrument type and performance to change from one study to another. Characteristics, such as the resolution of the analog-to-digital conversion, the sampling rate, the frequency range of flat instrument response, the full scale, and processing tasks such as filtering and resampling, may strongly affect the available range of frequencies in which $\kappa_{\mathrm{r}}$ is measured. Furthermore, the judgment of the analyst who selects the pertinent frequency range from $f_{1}$ to $f_{2}$ (Rovelli et al., 1988; Douglas et al., 2010), as well as the possible influence of the corner frequency within that frequency range, all introduce uncertainty into $\kappa_{0}$ estimates. Finally, the frequency range chosen may also bear upon the trade-off of $\kappa_{0}$ with the site amplification transfer function, whether using the classic approach coupled to the transfer function of the 1D soil column (Parolai and Bindi, 2004) or the response spectral approach coupled with the quarter-wavelength amplification of the generic profile (Boore and Joyner, 1997).

No study has compared all different available approaches in terms of $\kappa_{0}$. Some, however, have compared certain approaches with the classic approach. Edwards et al. (2011) compared the classic method with their broadband inversion, and Ktenidou et al. (2012) compared the classic with the transfer-function approach, both finding similar results, but with considerable scatter. Chandler et al. (2006) compared the classic method to that of response spectral fitting and found the latter approach overpredicted $\kappa_{0}$ values. Biasi and Anderson (2007) found that the displacement-based method gives an upper bound with respect to the classic method. Kilb et al. (2012) found similar though smaller overestimations using the displacement-based method. Ktenidou et al. (2013) found significant variation in $\kappa_{0}$ values when comparing different possible applications of the classic method.

To investigate the effect of the three aforementioned reasons behind the scatter of $\kappa_{0}-V_{S 30}$ correlations, we retrieve $\left(\kappa_{0}\right.$, $V_{S 30}$ ) data pairs from the literature and group them according to region and method. To the data collected by Van Houtte et al. (2011), we add recent results from Ktenidou et al. (2012, 2013) and Ktenidou and Van Houtte (2012), some of which use downhole data for the first time in $\kappa_{0}-V_{S 30}$ correlations. In Figure $2 \mathrm{~b}$, we group $\kappa_{0}-V_{S 30}$ data with region. Some separation is suggested between $\kappa_{0}$ values from stable continental (blue points) and active regions (all other colors), though the scatter is large. We then further separate data coming from Japan into two groups, based on the detailed $Q$ values presented in Oth et al. (2011). These authors divided Japan into four polygons with respect to $Q$ structure derived from crustal events (see their figs. 1 and 8 for details). Considering their $Q$ results at high frequencies, where $\kappa$ is computed, we group these polygons into a high- $Q$ zone (polygon 2 , with an average $Q$ ranging from 520 to 900 from 10 to $25 \mathrm{~Hz}$ ) and a low- $Q$ zone (polygons 1, 3, and 4 , with an average $Q$ 350 to 680 from 10 to $25 \mathrm{~Hz}$ ). We regress for these two zones separately and derive the two mean $\kappa_{0}-V_{S 30}$ correlations plotted in the figure as green lines. Indeed, $\kappa_{0}$ is on average $6 \mathrm{~ms}$ higher for the low- $Q$ zone (dark green) compared to the lower$Q$ zone (light green). Though this difference is rather small and lies within the scatter, this suggests the possibility that the 
underlying regional $Q$ variation may affect $\kappa_{0}$ for the same $V_{S 30}$. If so, such correlations may need to be used on a regional basis rather than a global one. We then group existing $\kappa_{0}-V_{S 30}$ data according to method. In Figure $2 \mathrm{c}$ we see that, especially for site classes $\mathrm{A}$ and $\mathrm{B}$, that is, soft and hard rocks, some of the lowest $\kappa_{0}$ values are predicted by the response spectrum and broadband approaches (blue, red), and some of the highest values are predicted by the classic approach (orange). It is worth noting that above $1500 \mathrm{~m} / \mathrm{s}$ the results of the acceleration spectrum (AS) approach are significantly similar for two very different regions: Greece and Switzerland (light green solid line and black dashed line of Fig. 2a). Conversely, for the Swiss data, two different approaches yield significantly different results (dashed black for AS and dark green for BB in Fig. 2a). This is an indication that the approach used may also have introduced a bias into the computation of $\kappa_{0}$ and hence $\kappa_{0}-V_{S 30}$ correlations for different approaches may not be directly comparable. Overall, we find effects of both region and method, but at this stage, it is difficult to dissociate them.

We stress once more that the scatter in $\kappa_{0}$ values due to region, method, and frequency range is only partly the reason behind the poor-correlation coefficients in most $\kappa_{0}-V_{S 30}$ correlations (usually less than 30\%). Another reason, as mentioned above, is the significant uncertainty in the estimation of $V_{S 30}$. Finally, another part of the problem is that $\kappa_{0}$ also relates to other physical parameters, such as, for example, the depth to bedrock, and probably more factors, which have not yet been mapped onto $\kappa_{0}$. Simply propagating the large scatter in $\kappa_{0}$ into ground-motion models, using the $\kappa$ correction process given for GMPE adjustments in Al Atik et al. (2014), would lead to a standard deviation of the $20 \mathrm{~Hz}$ spectral acceleration of 0.9 natural $\log$ units just due to $\kappa_{0}$ variability. This greatly exceeds the variability of high-frequency ground motions typically seen in empirical GMPE due to all sources of variability (source, site, and path), indicating that the $\kappa_{0}$ variability is correlated to other factors, which also affect the ground-motion level. Based on the above, reducing the existing inconsistencies arising from computation methods, regions, and ranges of frequencies will not solve the scatter issue, but it is a first step we need to take before we can start to decipher the physical basis of $\kappa_{0}$ and improve our methods of inferring it using more than just $V_{S 30}$. Finally, given the open question of the source component of $\kappa\left(\kappa_{\mathrm{s}}\right.$ of equation 1) and its possible contribution to the scatter, we believe that our suggestions toward consistency may help decipher these components (whether the source affects the scatter in $\kappa$ or even the median value) and better understand the remaining questions as to the physical interpretation of $\kappa$.

\section{TOWARD A MORE CONSISTENT ESTIMATION AND USE OF $\kappa$}

\section{Suggested Measurement Methods for Given Applications}

How should one measure site-specific, zero-distance $\kappa_{0}$ ? Within the scope of the measurement approaches and analyses suggested within the literature reviewed here, we suggest that certain measurement approaches may be more appropriate to use with certain applications. The general principle is that each measurement method uses a model with implicit assumptions about the effect of $\kappa$ on the spectrum, and those should be the same, or at least as similar as possible, to the assumptions made in the subsequent applications. We associate the suggested uses with the families of approaches defined in Table 1, taking into account the features of the approaches, such as, for example, the frequency band over which the measurement is performed.

- On the one hand, methods using the entire range of frequencies to compute $\kappa$ (such as $\kappa_{\mathrm{RESP}}$ and $\kappa_{\mathrm{BB}}$, that is, the fitting of simulated to observed response spectra and the broadband spectral inversion of Fourier spectra) may be better suited for use in stochastic ground-motion simulations. In such simulations, the $\kappa_{0}$ filter is often applied over the entire frequency band and not only at high frequencies. For instance, in Stochastic Model SIMulation (SMSIM; Boore, 2003), one of the most widely used codes for stochastic simulations, the diminution filter $e^{-\pi \kappa f}$ starts from $f_{1}=0$. In broadband simulations, the entire band is used to invert the Fourier spectrum for source, path, and site effects, whereas in any response spectrum the high frequencies are affected by the full frequency range of oscillators. The computation of $\kappa$ over the entire spectrum, given its known trade-offs with source parameters at lower frequencies, implies that the inversion results might best be interpreted relative to one another as a set of parameters rather than individually; this is also true for the group of parameters in a seismological model (Scherbaum et al., 2006). Boore et al. (1992) made a similar observation, noting that the $\kappa_{0}$ they computed with response spectral fitting for use in simulations might not correspond to the high-frequency classic $\kappa_{0}$ due to the difference in frequency ranges. EGFs may also be used to fix stress drop and avoid trade-offs, again within the notion of interdependability of the model parameters. For TGFs, there is also a trade-off between $\kappa$ and $Q$, which depends on how $Q$ is incorporated in the TGFs (frequency-dependent or independent $Q$ ).

- On the other hand, methods that derive $\kappa_{0}$ from direct measurements on high frequencies and distinctly separate it from regional attenuation (such as $\kappa_{\mathrm{AS}}, \kappa_{\mathrm{SS}}, \kappa_{\mathrm{IRVT}}$, and $\kappa_{\mathrm{TF}}$, i.e., the classic, source-spectrum, inverse random vibration theory, and transfer-function approaches) may tie better with adjustment of GMPEs from a host region (i.e., where the GMPE is well constrained by data) to a target region (i.e., a region where a GMPE is needed but little or no strong-motion data are available to constrain it). We suggest that the $\kappa_{\mathrm{AS}}, \kappa_{\mathrm{TF}}$, and $\kappa_{\mathrm{SS}}$ approaches may be most suitable for estimating host region $\kappa_{0}$ s (but also target region $\kappa_{0} \mathrm{~s}$, if appropriate data are available). In the case of $\kappa_{\text {IRVT }}$, we suggest it is by definition suitable for host regions, because it pertains to an existing GMPE.

- Finally, in what concerns the target regions in the host-totarget adjustment process, target $\kappa_{0} s$ are, at the moment, 
poorly constrained. These regions are often seismically inactive. Hence, the volume and magnitude range of available records does not generally allow use of the classic methods to compute $\kappa_{0}$. Furthermore, if $\kappa_{0}$ is to be inferred rather than measured, the uncertainty of existing $\kappa_{0}-V_{S 30}$ correlations poses a big problem. We suggest that, in the absence of any usable records at the site, $\kappa_{0}-V_{S 30}$ correlations may be used, recognizing the large uncertainty in the resulting $\kappa$ estimate. Correlations based on regional rather than global models should be preferred, for the reasons listed above. But overall, because even regional correlations do not describe $\kappa_{0}$ completely, sitespecific measurement of $\kappa_{0}$ rather than inference is strongly recommended. Thus, we suggest that the $\kappa_{\mathrm{DS}}$ approach is well worth investigating further, as it may help make use of very small magnitude events to make site-specific measurements of $\kappa_{0}$ in non-active regions.

To date, no study has undertaken to compare $\kappa_{0}$ values for the same data across all or most of the different measurement methods found in the literature, or examine the effects this may have on its use in existing applications and on the understanding of the underlying physics of $\kappa$ (though one attempt was made by Biasi and Anderson, 2007). We believe that such a study could bring to light inconsistencies in the approaches used today and could better demonstrate which $\kappa$ should be put to which use. Bearing in mind how multifaceted this parameter can be in both its meaning and its estimation, we suggest that when $\kappa$ values are measured, discussed, and used, they be accompanied by a notation similar to that shown in Table 1 . As $\kappa$ depends on the measurement approach and the underlying models, we believe these notations are needed to better suit the different purposes, as shown in the table.

\section{Suggestions on Instrumentation and Data Processing}

To the heterogeneity of the different approaches used, we may also add the possibility of constraints, problems, or errors in the available data or its processing. Ktenidou et al. (2013) show the effect of exceeding the available frequency range either as constrained by the signal-to-noise ratio (this should constrain $f_{2}$ ) or by the corner frequency (this should constrain $f_{1}$ ) in the estimation of $\kappa_{0}$, but the problem may begin even before this stage. For instance, Laurendeau et al. (2013) point out that, in choosing $f_{2}$ for their $\kappa_{\mathrm{AS}}$ estimation, Van Houtte et al. (2011) neglected to account for a low-pass filter present in all KiK-net instruments, which may have affected $\kappa_{\mathrm{r}}$. Data should preferably only be used after correcting for instrument response or, at least, checking the maximum usable frequency up to which response is flat. Similar problems may arise due not to the instrument but to the subsequent data processing protocol applied by data suppliers: Graizer (2012) demonstrates that standard procedures of filtering and resampling, which follow, for example, the Caltech protocol, may cause distortion to the Fourier and response spectra, affecting frequencies 6$8 \mathrm{~Hz}$ and above. He stresses the need for databases to supply original, unfiltered, un-corrected data in order to preserve high-frequency information in records. The above examples show that, although technological advances have allowed modern instruments to provide high sampling rates, certain standard practices do not allow us to take full advantage of data at high frequencies. This we believe is partly because we have been using such procedures due to momentum and partly because the interest of the engineering seismology community was focused until recently on lower-frequency response. As early as 1994, Trifunac (1994) pointed out that strong-motion processing tended to stop at $25 \mathrm{~Hz}$, even though it was already technologically possible to extend the limit to $50 \mathrm{~Hz}$. Such an extension would strongly benefit the study of $\kappa$. For instance, it would provide the frequency band necessary for the measurement of lower $\kappa_{\text {r_AS }}$ values on hard rock, like those coming from small nearby events or in stable regions with high $Q$. Going to higher frequencies is necessary to better compute very low values of $\kappa_{0}$, for example, at very hard rock sites, and stable regions, where considerable high-frequency energy is sometimes observed. It has been shown that the ability to measure $\kappa_{\mathrm{r}}$ for small nearby events depends partly on how far into the high frequencies $f_{2}$ can be extended (Ktenidou et al., 2013, fig. 4; Van Houtte et al., 2013). Today we are more aware of the importance of understanding ground motion at high frequencies, even above 30 or $50 \mathrm{~Hz}$; e.g., Silva and Darragh (2012), and Laurendeau et al. (2013) show the effect of $\kappa$ at frequencies above $50 \mathrm{~Hz}$. We also have the means to record and acquire data at higher frequencies. However, we need to be aware of pitfalls. For instance, in Japan, for some of the densest and highest-technology networks in the world, sampling rates are of 100 or $200 \mathrm{~Hz}$ (K-net and KiK-net, respectively). However, the instruments have a cutoff frequency of $30 \mathrm{~Hz}$ (Aoi et al., 2004), thus significantly limiting the usable band. We propose that future instrumentation initiatives take into account the need to improve knowledge at high frequencies and decide on sampling rates and anti-aliasing filters accordingly. This includes downhole arrays, which will help improve our understanding of hard-rock attenuation. Similarly, we suggest that public databases provide users with the option of direct access to uncorrected, unfiltered data, to avoid the problems outlined by Graizer (2012).

\section{CONCLUSION}

In this note, we have made four main suggestions, namely:

- that subscripts should be used when $\kappa_{0}$ is computed from data, so the user knows how the values were estimated,

- that certain families of $\kappa_{0}$ estimation approaches may be more appropriate for certain applications (Table 1),

- that rendering $\kappa_{0}-V_{S 30}$ correlations more consistent in terms of regions and methods may improve the current practice of inferring $\kappa_{0}$ without site-specific data and constitutes a useful step toward deciphering the physical basis of $\kappa_{0}$, and

- that future instrumentation and signal-processing protocols for open-access databases should take into account the preservation of high-frequency information. 
These recommendations notwithstanding, we stress that more research is needed to better comprehend this parameter and the scatter observed in its estimates. $\mathbf{<}$

\section{ACKNOWLEDGMENTS}

We are thankful for discussions with Philippe Renault and all partners and experts of the PEGASOS Refinement Project, through which the idea for this paper was born. We have also been inspired by Kilb et al. (2012), who named certain $\kappa$ estimation approaches, and comments by Pierre-Yves Bard. The research has been partly funded by the French SIGMA project. We thank Ralph Archuleta, Glenn Biasi, Jim Brune, Art Frankel, Tom Hanks, Maria Lancieri, Aurore Laurendeau, Walt Silva, Chris Van Houtte, and experts of Hanford SSHAC, SWUS SSHAC, Next Generation Attenuation-West 2 (NGA-W2), and SIGMA projects for useful discussions. Warm thanks go to Frank Scherbaum and John Douglas for insightful reviews of what was initially the "Don't call it kappa!" manuscript. Finally, we thank Dave Boore and the associate editor for thorough reviews that significantly improved the submitted manuscript. Some plots were made using Generic Mapping Tools v. 3.4 (www.soest.hawaii.edu/gmt, last accessed March 2013; Wessel and Smith, 1998).

\section{REFERENCES}

Abercrombie, R. E. (1995). Earthquake source scaling relationships from -1 to $5 M_{\mathrm{L}}$ using seismograms recorded at $2.5-\mathrm{km}$ depth, J. Geophys. Res. 100, 24,015-24,036.

Aki, K. (1967). Scaling law of seismic spectrum, J. Geophys. Res. 72, $1217-1231$.

Aki, K. (1987). Magnitude-frequency relation for small earthquakes: A clue to the origin of $f_{\max }$ of large earthquakes, J. Geophys. Res. 92, $1349-1355$.

Al Atik, L., A. Kottke, N. Abrahamson, and J. Hollenback (2014). Kappa correction of ground motion prediction equations using inverse random vibration theory approach, Bull. Seismol. Soc. Am. 104, no. 1, doi: $10.1785 / 0120120200$ (in press).

Anderson, J. G. (1986). Implication of attenuation for studies of the earthquake source, in (Maurice Ewing Series 6): Earthquake Source Mechanics, M. Ewing (Editor), American Geophysical Monograph 37 311-318.

Anderson, J. G. (1991). A preliminary descriptive model for the distance dependence of the spectral decay parameter in southern California, Bull. Seismol. Soc. Am. 81, 2186-2193.

Anderson, J. G., and S. E. Hough (1984). A model for the shape of the Fourier amplitude spectrum of acceleration at high frequencies, Bull. Seismol. Soc. Am. 74, 1969-1993.

Anderson, J. G., and J. R. Humphrey (1991). A least squares method for objective determination of earthquake source parameters, Seismol. Res. Lett. 62, 201-209.

Anderson, J. G., Y. Lee, Y. Zeng, and S. Day (1996). Control of strong motion by the upper 30 meters, Bull. Seismol. Soc. Am. 86, 1749-1759.

Aoi, S., T. Kunugi, and H. Fujiwara (2004). Strong-motion seismograph network operated by NIED: K-NET and KiK-net, J. Japan Assoc. Earthq. Eng. 4, 65-74.

Archuleta, R. J., E. Cranswick, C. Mueller, and P. Spudich (1982). Source parameters of the 1980 Mammoth Lakes, California, earthquake sequence, J. Geophys. Res. 87, 4595-4607.
Atkinson, G. M. (1996). The high-frequency shape of the source spectrum for earthquakes in eastern and western Canada, Bull. Seismol. Soc. Am. 86, 106-112.

Atkinson, G. M., and I. Beresnev (1997). Don't call it stress drop, Seismol. Res. Lett. 63, 3-4.

Bakun, W. H., C. G. Bufe, and R. M. Stewart (1976). Body wave spectra of central California earthquakes, Bull. Seismol. Soc. Am. 66, 439-459.

Beresnev, I. A., and G. M. Atkinson (1997). Modeling finite-fault radiation from the $\omega^{\mathrm{n}}$ spectrum, Bull. Seismol. Soc. Am. 87, 67-84.

Biasi, G. P., and J. G. Anderson (2007). Measurement of the parameter kappa, and reevaluation of kappa for small to moderate earthquakes at seismic stations in the vicinity of Yucca Mountain, Nevada, Final Technical Report TR-07-007, Nevada System of Higher Education (NSHE), University of Nevada, Las Vegas (UNLV), 232 pp., doi: $10.2172 / 920643$.

Biasi, G. P., and K. D. Smith (2001). Site Effects for Seismic Monitoring Stations in the Vicinity of Yucca Mountain, Nevada, MOL20011204.0045, a report prepared for the US DOE/ University and Community College System of Nevada (UCCSN) Cooperative Agreement.

Biro, Y., and P. Renault (2012). Importance and impact of host-to-target conversions for ground motion prediction equations in PSHA, in Proc. 15th World Conference of Earthquake Engineering, Lisbon, Portugal, 24-28 September, $10 \mathrm{pp}$.

Boore, D. M. (1986). Short-period $P$ - and $S$-wave radiation from large earthquakes: Implications for spectral scaling relations, Bull. Seismol. Soc. Am. 76, 43-64.

Boore, D. M. (2003). Simulation of ground motion using the stochastic method, Pure Appl. Geophys. 160, 635-676.

Boore, D. M. (2006). Determining subsurface shear wave velocities, $A$ review 3rd International Symposium on the Effects of Surface Geology on Seismic Motion, Grenoble, France, 30 August-1 September, Paper No. 103.

Boore, D. M., and W. B. Joyner (1997). Site amplifications for generic rock sites, Bull. Seismol. Soc. Am. 87, 327-341.

Boore, D. M., W. B. Joyner, and L. Wennerberg (1992). Fitting the stochastic omega-squared source model to observed response spectra in western North America: Trade-offs between stress drop and kappa, Bull. Seismol. Soc. Am. 82, 1956-1963.

Brune, J. (1970). Tectonic stress and the spectra of seismic shear waves, J. Geophys. Res. 75, 4997-5009.

Building Seismic Safety Council (BSSC) (2003). The 2003 NEHRP Recommended Provisions for New Buildings and Other Structures, Part 1: Provisions (FEMA 450), http://bssc.nibs.org (last accessed January 2013).

Campbell, K. W. (2003). Prediction of strong ground motion using the hybrid empirical method and its use in the development of ground motion (attenuation) relations in eastern North America, Bull. Seismol. Soc. Am. 93, 1012-1033.

Campbell, K. W. (2004). Erratum: Prediction of strong ground motion using the hybrid empirical method and its use in the development of ground motion (attenuation) relations in eastern North America, Bull. Seismol. Soc. Am. 94, 2418.

Campbell, K. W. (2009). Estimates of shear-wave $Q$ and $\kappa_{0}$ for unconsolidated and semiconsolidated sediments in eastern North America, Bull. Seismol. Soc. Am. 99, 2365-2392.

Castro, R., F. Pacor, A. Sala, and C. Petrungaro (1996). $S$ wave attenuation and site effects in the region of Friuli, Italy, J. Geophys. Res. 101, 22,355-22,369.

Chandler, A. M., N. T. K. Lamb, and H. H. Tsang (2006). Near-surface attenuation modelling based on rock shear-wave velocity profile, Soil Dynam. Earthq. Eng. 26, 1004-1014.

Cotton, F., F. Scherbaum, J. J. Bommer, and H. Bungum (2006). Criteria for selecting and adjusting ground-motion models for specific target regions: Application to Central Europe and rock sites, J. Seismol. 10, $137-156$. 
Delavaud, E., F. Cotton, S. Akkar, F. Scherbaum, L. Danciu, C. Beauval, S. Drouet, J. Douglas, R. Basili, M. Abdullah Sandikkaya, M. Segou, E. Faccioli, and N. Theodoulidis (2012). Towards a ground-motion logic tree for probabilistic seismic hazard assessment in Europe, J. Seismol. 16, 451-473.

Douglas, J., P. Gehl, L. F. Bonilla, and C. Gélis (2010). A $\kappa$ model for mainland France, Pure Appl. Geophys. 167, 1303-1315.

Drouet, S., F. Cotton, and P. Gueguen (2010). $V_{S 30}, \kappa$, regional attenuation and $M_{\mathrm{w}}$ from accelerograms: Application to magnitude 3-5 French earthquakes, Geophys. J. Int. 182, 880-898.

Edwards, B., D. Faeh, and D. Giardini (2011). Attenuation of seismic shear wave energy in Switzerland, Geophys. J. Int. 185, 967-984.

Fernández-Heredia, A. I., C. I. Huerta-Lopez, R. R. Castro-Escamilla, and J. Romo-Jones (2012). Soil damping and site dominant vibration period determination, by means of random decrement method and its relationship with the site-specific spectral decay parameter kappa, Soil Dynam. Earthq. Eng. 43, 237-246.

Foster, K. M., B. Halldorsson, R. A. Green, and M. C. Chapman (2012). Calibration of the specific barrier model to the NGA dataset, Seismol. Res. Lett. 83, 566-574.

Frankel, A. (1982). The effects of attenuation and site response on the spectra of microearthquakes in the northeastern Caribbean, Bull. Seismol. Soc. Am. 72, 1379-1402.

Frankel, A., D. Carver, E. Cranswick, M. Meremonte, T. Bice, and D. Overturf (1999). Site response for Seattle and source parameters of earthquakes in the Puget Sound region, Bull. Seismol. Soc. Am. 89, 468-483.

Gariel, J.-C., and M. Campillo (1989). The influence of the source on the high-frequency behaviour of the near-field acceleration spectrum: A numerical study, Geophys. Res. Lett. 16, 279-282.

Gentili, S., and G. Franceschina (2011). High frequency attenuation of shear waves in the southeastern Alps and northern Dinarides, Geophys. J. Int. 185, 1393-1416.

Ghofrani, H., G. Atkinson, and K. Goda (2013). Implications of the $2011 M 9.0$ Tohoku Japan earthquake for the treatment of site effects in large earthquakes, Bull. Earthq. Eng. 11, 171-203.

Graizer, V. (2012). Effect of low-pass filtering and re-sampling on spectral and peak ground acceleration in strong-motion records, Proc. 15th World Conference of Earthquake Engineering, Lisbon, Portugal, 24-28 September, 10 pp.

Graves, R. (1996). Simulating seismic wave propagation in 3D elastic media using staggered grid finite differences, Bull. Seismol. Soc. Am. 86, 1091-1106.

Graves, R. W., and A. Pitarka (2010). Broadband ground-motion simulation using a hybrid approach, Bull. Seismol. Soc. Am. 100, 2095-2123.

Halldorsson, B., and A. S. Papageorgiou (2005). Calibration of the specific barrier model to earthquakes of different tectonic regions, Bull. Seismol. Soc. Am. 95, 1276-1300.

Hanks, T. C. (1982). $f_{\max }$, Bull. Seismol. Soc. Am. 72, 1867-1879.

Hanks, T. C., and R. K. McGuire (1981). The character of high-frequency strong ground motion, Bull. Seism. Soc. Am. 71, 2071-2095.

Hiemer, S., F. Scherbaum, D. Roessler, and N. Kuehn (2011). Determination of $\tau_{0}$ and rock site $\kappa$ from records of the 2008/2009 earthquake swarm in western Bohemia, Seismol. Res. Lett. 82, 387-393.

Hough, S. E. (1997). Empirical Green's function analysis: Taking the next step, J. Geophys. Res. 102, 5369-5384.

Hough, S. E., and J. G. Anderson (1988). High-frequency spectra observed at Anza, California: Implications for $Q$ structure, Bull. Seismol. Soc. Am. 78, 692-707.

Hough, S. E., J. G. Anderson, J. Brune, F. Vernon III, J. Berger, J. Fletcher, L. Haar, T. Hanks, and L. Baker (1988). Attenuation near Anza, California, Bull. Seismol. Soc. Am. 78, 672-691.

Hough, S. E., J. Lees, and F. Monastero (1999). Attenuation and source properties at the Coso geothermal area, California, Bull. Seismol. Soc. Am. 89, 1606-1619.

Humphrey, J. R., Jr., and J. G. Anderson (1992). Shear wave attenuation and site response in Guerrero, Mexico, Bull. Seismol. Soc. Am. 81, $1622-1645$.
Irikura, K. (1986). Prediction of strong acceleration motion using empirical Green's function, Proc. 7th Japan Earthq. Eng. Symp., 10-12 December 1986, Tokyo, 151-156.

Iwakiri, K., and M. Hoshiba (2012). High-frequency (>10 Hz) content of the initial fifty seconds of waveforms from the 2011 Off the Pacific Coast of Tohoku Earthquake, Bull. Seismol. Soc. Am. 102, 2232-2238.

Kilb, D., G. Biasi, J. G. Anderson, J. Brune, Z. Peng, and F. L. Vernon (2012). A comparison of spectral parameter kappa from small and moderate earthquakes using southern California ANZA seismic network data, Bull. Seismol. Soc. Am. 102, 284-300.

Ktenidou, O.-J., and C. Van Houtte (2012). Empirical Estimation of kappa from Rock Velocity Profiles at the Swiss NPP Sites (19.02.2012), Report TP2-TB-1090, PEGASOS Refinement Project.

Ktenidou, O.-J., S. Drouet, N. Theodulidis, M. Chaljub, S. Arnaouti, and F. Cotton (2012). Estimation of kappa $(\kappa)$ for a sedimentary basin in Greece (EUROSEISTEST): Correlation to site characterization parameters, Proc. 15th World Conference of Earthquake Engineering, Lisbon, Portugal, 24-28 September, 10 pp.

Ktenidou, O.-J., C. Gelis, and F. Bonilla (2013). A study on the variability of kappa in a borehole, Implications on the computation method used, Bull. Seismol. Soc. Am. 103, 1048-1068.

Lancieri, M., R. Madariaga, and F. Bonilla (2012). Spectral scaling of the aftershocks of the Tocopilla 2007 earthquake in northern Chile, Geophys. J. Int. 189, 469-480.

Laurendeau, A., F. Cotton, O. -J. Ktenidou, L -F. Bonilla, and F. Hollender (2013). Rock and stiff-soil site amplification: Dependencies on $V_{S 30}$ and kappa $\left(\kappa_{0}\right)$, Bull. Seismol. Soc. Am. 103, no. 6, doi: $10.1785 / 0120130020$.

Mai, P. M., W. Imperatori, and K. B. Olsen (2010). Hybrid broadband ground-motion simulations: Combining long-period deterministic synthetics with high-frequency multiple $\mathrm{S}$-to-S back-scattering, Bull. Seismol. Soc. Am. 100, 2124-2142.

Margaris, B. N., and D. M. Boore (1998). Determination of $\Delta \sigma$ and $\kappa_{0}$ from response spectra of large earthquakes in Greece, Bull. Seismol. Soc. Am. 88, 170-182.

Mayeda, K., and W. Walter (1996). Moment, energy, stress drop, and source spectra of western United States earthquakes from regional coda envelopes, J. Geophys. Res. 101, 11,195-11,208.

Moss, R. E. S. (2008). Quantifying measurement uncertainty of thirtymeter shear-wave velocity, Bull. Seismol. Soc. Am. 98, 1399-1411.

Motazedian, D. (2006). Region-specific key seismic parameters of earthquakes in northern Iran, Bull. Seismol. Soc. Am. 96, 1383-1395.

Nava, F. A., R. García, R. R. Castro, C. Suárez, B. Márquez, F. Núñez-Cornú, G. Saavedra, and R. Toscano (1999). $S$ wave attenuation in the coastal region of Jalisco-Colima, Mexico, Phys. Earth Planet. In. 115, 247-257.

Oth, A., D. Bindi, S. Parolai, and D. Di Giacomo (2011). Spectral analysis of K-NET and KiK-net data in Japan, Part II: On attenuation characteristics, source spectra, and site response of borehole and surface stations, Bull. Seismol. Soc. Am. 101, 667-687.

Papageorgiou, A. S. (1988). On two characteristic frequencies of acceleration spectra: Patch corner frequency and $f_{\max }$, Bull. Seismol. Soc. Am. 78, 509-529.

Papageorgiou, A. S. (2003). The barrier model and strong ground motion, Pure Appl. Geophys. 160, 603-634.

Papageorgiou, A. S., and K. Aki (1983). A specific barrier model for the quantitative description of inhomogeneous faulting and the prediction of strong ground motion. Part I: Description of the model, Bull. Seismol. Soc. Am. 73, 693-722.

Parolai, S., and D. Bindi (2004). Influence of soil-layer properties on $k$ evaluation, Bull. Seismol. Soc. Am. 94, 349-356.

Purvance, M. D., and J. G. Anderson (2003). A comprehensive study of the observed spectral decay in strong-motion accelerations recorded in Guerrero, Mexico, Bull. Seismol. Soc. Am. 93, 600-611.

Rathje, E. M., A. R. Kottke, and M. C. Ozbey (2005). Using inverse random vibration theory to develop input Fourier amplitude spectra 
for use in site response, Proc. 16th Int. Conf. Soil Mechanics and Geotechnical Engineering: TC4 Earthquake Geotechnical Engineering Satellite Conf., 10 September 2005, Osaka, Japan, 160-166.

Rovelli, A., O. Bonamassa, M. Cocco, M. Di Bona, and S. Mazza (1988). Scaling laws and spectral parameters of the ground motion in active extensional areas in Italy, Bull. Seismol. Soc. Am. 78, 530-560.

Scherbaum, F. (1990). Combined inversion for the three-dimensional $Q$ structure and source parameters using microearthquake spectra, J. Geophys. Res. 95, 12,423-12,438.

Scherbaum, F., F. Cotton, and H. Staedtke (2006). The estimation of minimum-misfit stochastic models from empirical ground-motion prediction equations, Bull. Seismol. Soc. Am. 96, 427-445.

Schmedes, J., R. J. Archuleta, and D. Lavallée (2010). Correlation of earthquake source parameters inferred from dynamic rupture simulations, J. Geophys. Res. 115, no. B03304, doi: 10.1029/2009JB006689.

Silva, W. J., N. Abrahamson, G. Toro, and C. Costantino (1997). Description and Validation of the Stochastic Ground Motion Model, Report submitted to Brookhaven National Laboratory, Associated Universities, Inc., Upton, New York 11973, Contract No. 770573.

Silva, W. J., and R. Darragh (1995). Engineering Characterization of Earthquake Strong Ground Motion Recorded at Rock Sites, Palo Alto, Electric Power Research Institute, TR-102261.

Silva, W. J., and R. Darragh (2012). Assessment of kappa for Vertical and Horizontal Motions at Rock Sites Using Spectral Shapes, Final Report no. EXT-TB-1089, Pegasos Refinement Project.

Silva, W., R. Darragh, N. Gregor, G. Martin, N. Abrahamson, and C. Kircher (1998). Reassessment of Site Coefficients and Near-Fault Factors for Building Code Provisions, Technical Report Program Element II: 98-HQGR-1010, Pacific Engineering and Analysis, El Cerrito, U.S.A.

Toro, G. R., N. A. Abrahamson, and J. F. Schneider (1997). Model of strong ground motions from earthquakes in central and eastern North America: Best estimates and uncertainties, Seismol. Res. Lett. 68, 41-57.

Trifunac, M. D. (1994). $Q$ and high-frequency strong motion spectra, Soil Dynam. Earthq. Eng. 13, 149-161.

Tsai, C.-C. P., and K.-C. Chen (2000). A model for the high-cut process of strong motion accelerations in terms of distance, magnitude, and site condition: An example from the SMART 1 array, Lotung, Taiwan, Bull. Seismol. Soc. Am. 90, 1535-1542.

Tsurugi, M., T. Kawaga, and K. Irikura (2008). Study on high-cut frequency characteristics of ground motions for inland crustal earthquakes in Japan, Proc. 14th WCEE, Beijing, 12-17 October.
Van Houtte, C., S. Drouet, and F. Cotton (2011). Analysis of the origins of $\kappa$ (kappa) to compute hard rock to rock adjustment factors for GMPEs, Bull. Seismol. Soc. Am. 101, 2926-2941.

Van Houtte, C., O. -J. Ktenidou, and T. Larkin (2013). Near-source kappa estimates from the Canterbury earthquake sequence, New Zealand, SSA Annual Meeting, Salt Lake City, 17-19 April 2013.

Wen, J., and X. Chen (2012). Variations in $f_{\max }$ along the ruptured fault during the $M_{\mathrm{w}} 7.9$ Wenchuan earthquake of 12 May 2008, Bull. Seismol. Soc. Am. 102, 991-998.

Wessel, P., and W. H. F. Smith (1998). New, improved version of the Generic Mapping Tools Released, Eos Trans. $A G U$ 79, 579.

Zeng, Y., J. G. Anderson, and G. Yu (1994). A composite source model for computing realistic synthetic strong ground motions, J. Res. Lett. 21, $725-728$.

Olga-Joan Ktenidou Fabrice Cotton ISTerre Université de Grenoble 1 CNRS, F-38041 Grenoble France

olga.ktenidou@ujf-grenoble.fr

\author{
Norman A. Abrahamson \\ Department of Civil and Environmental Engineering \\ University of California, Berkeley \\ 447 Davis Hall \\ Berkeley, California 94720 U.S.A.
}

John G. Anderson

Nevada Seismological Laboratory

and Department of Geological Sciences and Engineering

MS 174

University of Nevada

Reno, Nevada 89557 U.S.A. 